\title{
Recognizing Recreational Water Exposure and Habituating HAB Surveillance in ESSENCE
}

\author{
Kathryn Kuspis*, Meredith Jagger, Melissa Powell and Rebecca Hillwig
}

Public Health Division, Oregon Health Athority, Portland, OR, USA

\section{Objective}

Use ESSENCE to create a sustainable process for identifying ED and urgent care visits that may be related to harmful algal bloom exposure in Oregon.

\section{Introduction}

Harmful algal blooms (HABs) consist of colonies of prokaryotic photosynthetic bacteria algae that can produce harmful toxins. The toxins produced by HABs are considered a One Health issue. HABs can occur in all types of water (fresh, brackish, and salt water) and are composed of cyanobacteria or microalgae. As the climate changes, so do many of the factors that contribute to the growth of HABs, which in turn, can increase the incidence of HAB-related illness in humans.

There are three main pathways that HAB toxins can affect human health: dermal, gastrointestinal (GI), and neurological. Swimming in or consuming contaminated water and eating contaminated shellfish are ways to develop HAB-related illnesses. Contact with cells from a bloom while recreating can cause a rash on the body. Most commonly, HAB-related illnesses present with GI symptoms that resemble food poisoning and can affect the liver. Rarely, HABs that produce cyanotoxins can present with neurological symptoms.

Issuing and lifting freshwater $\mathrm{HAB}$ advisories is within the preview of the Environmental Public Health section at the Oregon Public Health Division. However, most water bodies in the state are not monitored. Because of this, syndromic surveillance was considered as a potentially useful source of HAB exposure information, and the Oregon ESSENCE team was asked to develop a query to help monitor HAB-related complaints.

\section{Methods}

Preliminary research was done on HABs and the associated health issues, and past advisories were examined to identify locations of interest. Next, keywords and symptoms were evaluated.

Initially, the objective was to create a single query for $\mathrm{HAB}$ syndromic surveillance, but it became evident that multiple queries would have to be developed to fully encompass the various types of HAB-related illnesses: GI, neurological, and rash.

Most commonly Oregon ESSENCE uses chief complaint and discharge diagnosis (CCDD) queries. However, the ICD-10 codes relating to HABs are not widely used, with only two occurrences since June 2015. It was determined that using the already established ESSENCE syndromes of Neuro, GI, and Rash would be most useful. To make the queries HAB-specific, an additional exposure element needed to be added. Exposures to HABs that are of interest occur in recreational freshwater sources. After running this query in the CCDD field, it was determined that the triage note field would yield better results. This is because this field often includes the patient's verbatim complaints. This produced higher quality results, and a seasonal curve of cases could be seen in the historic data.

Since the microcystin threshold for illness is significantly lower for pets; and a permanent $\mathrm{HAB}$ alert in southern Oregon was established after several dogs died from drinking contaminated water, tracking neurological cases that followed canine illness was investigated. A free-text triage note query was developed for patients mentioning dogs, and it was combined with the ESSENCE Neuro syndrome.
After several attempts, it was clear that this would not be helpful for surveillance of HAB-related illnesses.

Ultimately, four query configurations were developed to monitor HAB-related illness. Most importantly, a free-text recreational water query was developed to stand alone and then be paired with three distinct ESSENCE syndromes.

Recreational water query text: $\left(,\left(, \wedge\right.\right.$ lake ${ }^{\wedge}$, andnot, $\left(, \wedge^{\wedge} \operatorname{road}^{\wedge}\right.$, or, $\wedge^{\wedge} \mathrm{rd}^{\wedge}$, or, $\wedge^{\wedge} \mathrm{sky}^{\wedge}$, or, $\wedge^{\wedge}$ oswego $0^{\wedge}$, or, $\wedge^{\wedge}$ view $\left.\left.^{\wedge},\right),\right)$, or,,${ }^{\wedge}$ swim $^{\wedge}$, or, $\left(,{ }^{\wedge}\right.$ river $\wedge$, andnot, $\left(, \wedge^{\wedge}\right.$ driver ${ }^{\wedge}$, or, $\wedge^{\wedge} \operatorname{hood}^{\wedge}$, or, ${ }^{\wedge} \mathrm{rd}^{\wedge}$, or, ${ }^{\wedge} \operatorname{road}^{\wedge}$, or, ${ }^{\wedge}$ three $\left.{ }^{\wedge},\right)$, , or, ${ }^{\wedge}$ boat $^{\wedge}$, ), andnot, ${ }^{\wedge}$ feels like ${ }^{\wedge}$

All queries were compiled into a myESSENCE page that could be shared for easy monitoring by all members of the team (Figure 1).

\section{Results}

The ESSENCE team monitored the HAB myESSENCE page. The monitoring period for this project stretched from May to early August (MMWR weeks 19-31). Motoring was often informed by HAB alerts and required looking closely at individual visits. Over this time, the number of recreational water related visits varied, but the average was approximately 110 visits a week. This techniques also helped identify cases possibly related to unreported blooms. The months of June and July saw 15 specific cases that were potentially due to HAB exposure. These cases were highlighted and forwarded to Environmental Public Health for investigation.

\section{Conclusions}

This process helped refine the use of the triage note field when constructing keyword queries. While not all Oregon facilities provide triage notes, using specific terms allows ESSENCE users to search for words that may not be included in chief complaints. This is most useful when searching for specific places or events. With further analysis, users can see what chief complaints are most likely to occur in conjunction with specific exposures. Moving forward, the development of a recreational water query has proven to be useful beyond the scope of this HAB project. Alternative versions of this query have been used in other contexts.

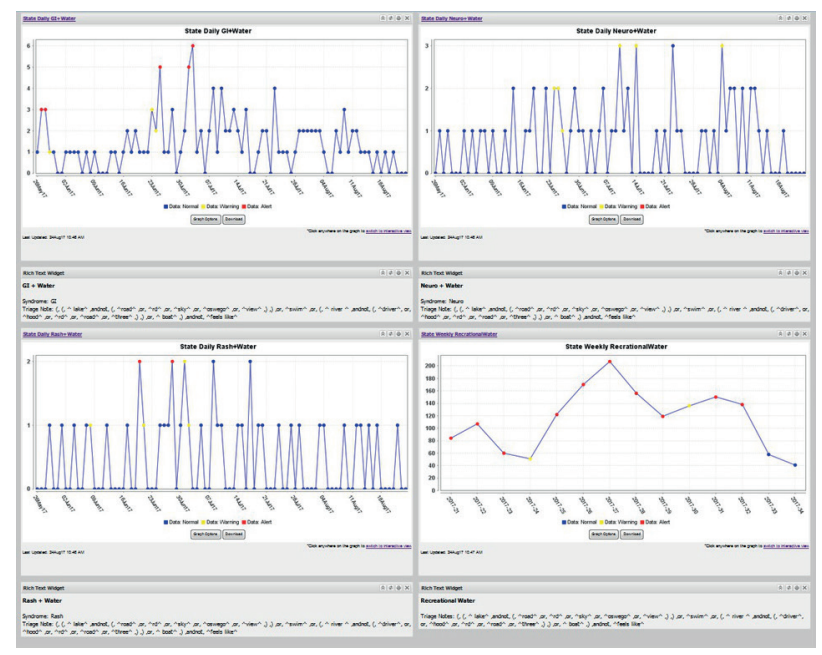




\section{ISDS 2018 Conference Abstracts}

\section{Keywords}

HAB; Harmful agal bloom; ESSENCE; Surveillance; Recreational water

\section{References}

Harmful Algal Bloom (HAB)-Associated Illness. (2017, June 01). Retrieved August 01, 2017, from https://www.cdc.gov/habs/index. html

\section{*Kathryn Kuspis}

E-mail: kathryn.a.kuspis@state.or.us 